American Journal of Environmental Sciences 8 (2): 143-151, 2012

ISSN 1553-345X

(C) 2012 Science Publications

\title{
Effect of Dose-Response of Zinc and Manganese on Siderophores Production
}

\author{
${ }^{1}$ Mehri Ines, ${ }^{1}$ Khessairi Amel, ${ }^{1}$ Turki Yousra, \\ ${ }^{1}$ Saidi Neila, ${ }^{1}$ Daly Imen, ${ }^{2}$ Meyer Jean Marie and ${ }^{1}$ Hassen Abdennasseur \\ ${ }^{1}$ Laboratory of Treatment and Water Recycling, \\ Centre of Research and Water Technologies, \\ Technopark of Borj-Cedria, Tunisia \\ ${ }^{2}$ Laboratoiratory of Microbiology and Genetic, \\ University of Louis-Pasteur, CNRS FRE 2326, Strasbourg, France
}

\begin{abstract}
Problem statement: This study was conducted to find and determine whether the siderophores of the four environmental Pseudomonas spp. isolates possess a sequestering activity towards essential transition metals ( $\mathrm{Zn}$ and $\mathrm{Mn}$ ) other than iron. Approach: Four fluorescent Pseudomonads isolated from various environments, were characterized analytically (Isoelectric focusing), biologically (pyoverdine-mediated uptake) and genetically (16S rDNA sequencing). By means of spectrophotometric measurements, it was possible to establish and compare the levels of pyoverdine production, in two different nutrient-poor media. Results: The strains were assigned, by sequencing, to $P$. fluorescens, $P$. aeruginosa, $P$. putida and $P$. mosselii isolated, respectively from soil, compost, sea water and waste water treatment plant. These bacterial strains were recognized as producing diver's yellow-green siderophores types, when grown under conditions of iron starvation. The highest metabolite concentration was obtained with PsC132 and PsTp171 strains isolated respectively from compost and waste water treatment plant, in CAA medium. Strains grown in CAA medium exhibit a higher PVD level compared to SM medium. Mn (II) was found to promote pyoverdine biosynthesis, but rather, $\mathrm{Zn}$ (II) had no significant effect on siderophore production when compared to control medium. For both strains PsS29 and PsC132, the increase of iron concentration quenched siderophore production especially above $20 \mu \mathrm{M}$. Pyoverdine level declined with the high concentration of zinc but increased with Manganese concentration ranging up to $70 \mu \mathrm{M}$ (in case of PsC132) and $300 \mu \mathrm{M}$ (in case of PsS29). Conclusion/Recommendations: The ability of fluorescent Pseudomonas, isolated from wastewater treatment plant and from compost, to sequester zinc, point to a unique advantage of these species for divers bioremediation applications.
\end{abstract}

Keywords: Fluorescent pseudomonads, heavy metals, iron-poor media, siderophore production, bacterial strains, control medium, bacterial strains, non-proliferating conditions

\section{INTRODUCTION}

A number of transition metals are needed by bacteria as vital constituents, but their availability in the environment may not suffice to support microbial growth state (Ambrosi et al., 2002; Adarsh et al., 2007). Some authors found that production of pyoverdines contributes to the bio-control capacity of the fluorescent Pseudomonas. Conversely, other studies failed to establish a link between production of pyoverdine and antagonism against phytopathogenic fungi Kumar et al.,2008; Saidi et al., 2009; Yang et al., 2009.
Pseudomonas spp. has been shown to produce siderophores able to chelate any available iron (Henry et al., 1991). Due to the critical need for iron in aerobic metabolism, bacteria living in neutral environments are normally faced to the nutritional iron deficit resulting from the low solubility of iron in its oxidized state (Winkelmann et al., 1987; Ambrosi et al., 2002). In order to satisfy their need to iron, microorganisms start to excrete large amounts of specific $\mathrm{Fe}^{3+}$ scavenging molecules (siderophores), when cells are grown under iron deficiency (Braun and Braun, 2002). The Fe (III)siderophore complex is then transported into bacterial cell via cognate-specific receptor to enzymatic

Corresponding Author: Mehri Ines, Laboratory of Treatment and Water Recycling, Centre of Research and Water Technologies, Technopark of Borj-Cedria, Tunisia 
reduction (Meyer et al., 2000; Cornelis and Matthijs, 2002). Pyoverdine (PVD), the fluorescent siderophore produced by the rRNA groupI species of genus Pseudomonas, constitutes a large family of iron chelators (Wahyudi et al., 2011). This Yellow-green fluorescent pigment is composed of three structural parts: dihydroxyquinoline chromophore responsible of the fluorescence, a variable peptide part comprising 6 to 12 amino acids and a side chain, generally a dicarboxylic acid or a dicarboxylic amid (Meyer et al., 2000; Cornelis and Matthijs, 2002). As the peptide part interacts with specific cell surface receptors, pyoverdine type recognition allows for Pseudomonas strain classification (Meyer et al., 2002).

Some siderophores can bind, besides iron, other essential metals $(\mathrm{K}, \mathrm{Mg}$,) that serve as micronutrients, used for redox processes and regulation of osmotic pressure (Visca et al., 1992; Bruins et al., 2000; Parker et al., 2004; Shinozaki-Tajiri et al., 2004). Moreover, microorganisms able to produce siderophores can protect themselves by binding toxic metals $(\mathrm{Al}, \mathrm{Pb}$, Cd,) (Mureseanu et al., 2003; Olmo et al., 2003).

Although essential metals have important biological role, at high levels they can damage cell membranes, alter enzyme specificity, disrupt cellular functions, damage the DNA structure (Bruins et al., 2000; Canovas et al., 2003; Teitzel et al., 2006) and can reduce crop yields and soil fertility (Stuczynski et al., 2003).

The objectif of the research was to evaluate environmental Pseudomonas spp in siderophores sequestering activity towards essential transition metals ( $\mathrm{Zn}$ and $\mathrm{Mn}$ ) other than iron. The effect of pyoverdin production was studied in two metal poor mediums (succinate and casamino Acid). The dose-response effect of Fe (III), Zn (II) and Mn (II) was then tested on siderophore production.

\section{MATERIALS AND METHODS}

Isolation of Pseudomonas fluorescent strains: In this study the bacterial strains were isolated from various media: soil (PsS29), compost (PsC132), sea water (PsWs140) and waste water treatment plant (PsTp171).

Fluorescent pseudomonad colonies were isolated on King's B medium (Scharlau) and identified under UV light at $366 \mathrm{~nm}$. Purified single colonies were further spread onto KB agar plates to obtain pure cultures. Stock cultures were made in Luria Bertani broth containing $50 \%(\mathrm{w} / \mathrm{v})$ glycerol and stored at $-80^{\circ} \mathrm{C}$.

IEF analysis and PVD-mediated iron uptake: The iron-poor liquid Casamino Acid (CAA) growth medium used for this study was composed as follows (per liter): $5 \mathrm{~g}$ of low-iron Bacto Casamino Acid (Difco), $1.54 \mathrm{~g}$ of $\mathrm{K}_{2} \mathrm{HPO}_{4} \cdot 3 \mathrm{H}_{2} \mathrm{O}$ and $0.25 \mathrm{~g}$ of $\mathrm{MgSO}_{4} \cdot 7 \mathrm{H}_{2} \mathrm{O}$. CAA medium was mainly used for PVD-IEF analysis and
PVD purification through the Amberlite XAD-4 (XAD) procedure as previously described by Meyer et al. (2002). The cultures were incubated on a rotary shaker $(200 \mathrm{rpm})$ at $25^{\circ} \mathrm{C}$. The model 111 mini-IEF cell from Bio-Rad was used. Casting of the gels $(5 \%$ polyacrylamide containing $2 \%$ Bio-Lyte $3 / 10$ ampholytes) and electric focusing were performed according to the manufacturer's recommendations. One-microliter samples of PVDs (aqueous XADpurified solutions $\left(6.5 \mathrm{mg} \mathrm{mL} \mathrm{m}^{-1}\right)$ ), or of culture supernatants (40-h CAA-grown culture supernatant concentrated 20 -fold by lyophilisation) were used in this experiment. PVD bands in the gel were visualized under UV light at $365 \mathrm{~nm}$ and photographed immediately after focusing. Their respective isoelectric $\mathrm{pH}$ values (pHi values) were determined with "Easy win 32" program as described by Fuchs et al. (2001). This allowed assigning each band to the corresponding pHi value. A mixture of seven known pyoverdines bands (3.95, 4.6, 5.2, 7.25, 7.75, 8.8 and 9.2) was used as internal pHi standard (Meyer et al., 2002).

The PVD-mediated iron uptake analysis was conducted as previously described by Meyer et al. (2002). Iron-starved cells were then incubated in succinate medium under non-proliferating conditions in the presence of a label mix containing ${ }^{59} \mathrm{Fe}-\mathrm{PVD}$ complex. Aliquots of the bacterial suspension were withdrawn at different time intervals and rapidly filtered on $0.45 \mu \mathrm{m}$ porosity membrane. Cells remaining on the filters were thoroughly washed and their radioactivity, measuring the amount of label iron incorporated during the incubation time, was determined using a Gamma 4000 Beckman radioactivity counter. Control assays without bacteria were performed simultaneously to verify the complete solubility of labelled iron through PVD complexation.

Partial sequencing of the 16S rDNA gene: PCR amplifications were performed using the following primers: forward primer Ps-for (5'GGTCTGAGAGGATGATCAGT-3') and reverse primer Ps-rev (5'-TTAGCTCCACCTCGCGGC-3') for $16 \mathrm{~S}$ rRNA gene.

The 16S rRNA gene PCR products were purified using the QIAquick Wizard PCR purification Kit (Promega, USA), according to manufacturer's instructions. Sequences of the PCR products obtained with Ps-for/Ps-rev primers were aligned and corrected manually with Chromas Pro (version 1.34). Similarity matrix of $16 \mathrm{~S}$ rRNA gene sequences with closest neighbours and identification were achieved using RDP utilities (Ribosomal Database Project II: http://rdp.cme.msu.edu/html). 
Am. J. Environ. Sci., 8 (2): 143-151, 2012

Pyoverdine assay: The media used in this investigation were the King's $\mathrm{B}$, the succinate medium ( $\mathrm{SM}, \mathrm{g} \mathrm{L}^{-1}$ : $\mathrm{K}_{2} \mathrm{HPO}_{4}, 6.0 ; \mathrm{KH}_{2} \mathrm{PO}_{4}, 3.0 ; \mathrm{MgSO}_{4} \cdot 7 \mathrm{H}_{2} \mathrm{O}, 0.2$; $\left(\mathrm{NH}_{4}\right)_{2} \mathrm{SO}_{4}, 1.0$; Succinic acid, 4.0; pH 7.0) and Casamino Acid media (CAA, g L ${ }^{-1}: \mathrm{K}_{2} \mathrm{HPO}_{4} \cdot 3 \mathrm{H}_{2} \mathrm{O}$, 1.54; $\mathrm{MgSO}_{4} \cdot 7 \mathrm{H}_{2} \mathrm{O}, 0.25$; Bacto Casamino Acid (Difco), 5.0; pH 7.0).

All these media were prepared with deionized water. To prevent siderophore interaction with other elements, glassware was cleaned in $6 \mathrm{M} \mathrm{HCl}$ and repeatedly rinsed with ultrapure water.

The culture broth was inoculated with actively grown culture ( $16 \mathrm{~h}$ in King's B medium) and grown in $60 \mathrm{~mL}$ of iron deficient succinate broth and CAA media at $28^{\circ} \mathrm{C}$ for $48 \mathrm{~h}$ under constant shaking of $150 \mathrm{rpm}$ using an incubator shaker (ZHWY-2102 P). Samples of $1 \mathrm{~mL}$ were taken at 5, 10, 24, 36 and $48 \mathrm{~h}$ interval times respectively. Bacterial growth was estimated by spectrophotometry at $600 \mathrm{~nm}$. The culture broth were then withdrawn and centrifuged at 10,000 rpm for 15 min at $4^{\circ} \mathrm{C}$. Decimal dilutions of supernatants were done in deionised water. The amount of siderophores excreted into the culture medium was determined by spectrophotometry at $405 \mathrm{~nm}$ (Spectro UVS-2700 Dual BEAM LABOMED, INC) in $1 \mathrm{~cm}$ cells against media blank. Pyoverdin levels were expressed as the ratio of A405/A600 (Stintzi et al., 2000). Three repeated experiments were envisaged.

Effect of metal concentration on growth and pyoverdine production: Metal Salts were used in the following forms: $\mathrm{ZnSO}_{4} \cdot 7 \mathrm{H}_{2} \mathrm{O}$ (iron content, <10 ppm); $\mathrm{MnSO}_{4} \cdot \mathrm{H}_{2} \mathrm{O}$ (iron content, <0.001\%) and FeSO4. $7 \mathrm{H}_{2} \mathrm{O}$ (iron content, >99.9\%). Stocks of $10 \mathrm{mM}$ $\mathrm{ZnSo}_{4}$ and $\mathrm{MnSo}_{4}$ salts were prepared and sterilized with $0.22 \mu \mathrm{m}$ filters under aseptic condition. These stock solutions were incorporated in autoclaved CAA media $(\mathrm{CAA}+\mathrm{Zn}$ and $\mathrm{CAA}+\mathrm{Mn})$ and $\mathrm{SM}$ media $(\mathrm{SM}$ $+\mathrm{Zn}$ and $\mathrm{SM}+\mathrm{Mn}$ ) at a final concentration of $60 \mu \mathrm{M}$ for each metal.

In order to determine the threshold level of metals at which growth and/or siderophore biosynthesis are stimulated or repressed, PsS29 and PsC132 strains in CAA medium were monitored as a function of increasing amounts of $\mathrm{Fe}$ (III), $\mathrm{Zn}$ (II) and $\mathrm{Mn}$ (II) from $0.1-475 \mu \mathrm{M}$ in $5 \mathrm{~mL}$ CAA medium.

\section{RESULTS}

Strain characterisation: In an attempt to assign isolates to bacterial species, the strains referenced as PsS29, PsC132, PsWs140 and PsTp171 and selected in this investigation, showed a $16 \mathrm{~S}$ rDNA sequence related to four Pseudomonas species, namely: $P$. fluorescens, $P$. aeruginosa, $P$. putida and $P$. mosselii, respectively.

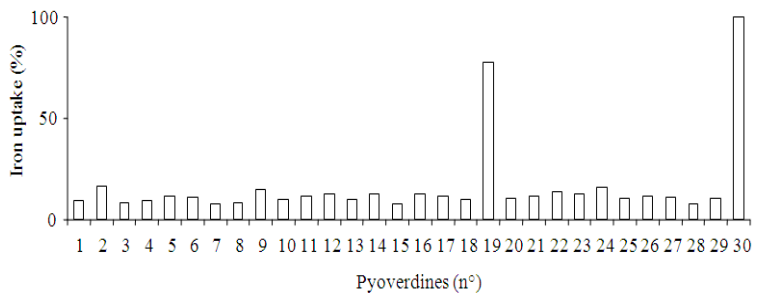

Fig. 1: Homologous and heterologous PVD-mediated ${ }^{59} \mathrm{Fe}$ incorporation by PsWs. 140 strain. Ordinate values correspond to ${ }^{59} \mathrm{Fe}$-radioactivity incorporated into the cells expressed in percentage, with $100 \%$ representing the incorporation obtained when using the homologous pyoverdine as ${ }^{59} \mathrm{Fe}$-iron chelator (pyoverdine number 30 in abscissa). The other pyoverdines tested in abscissa (numbers 1 to 29 as alkaline pyoverdines correspond to the structurally different pyoverdines synthesized by the following bacterial strains: 1 : Pseudomonas sp. G24, 2: Pseudomonas sp. G83, 3: Pseudomonas sp. G84, 4: Pseudomonas sp. G169, 5: Pseudomonas sp. CFML 96-312, 6: Pseudomonas sp. CFML 96-318, 7: Pseudomonas sp. CFML 95-275, 8: Lille25, 9: Pseudomonas sp. strain E8, 10: P.fluorescens SB8.3, 11: Pseudomonas sp. A6, 12: P.fluorescens PL8, 13: P.Kilonensis, 14: P.fluorescens ATCC 13525, 15: P.fluorescens 18.1, 16: P.aeruginosa PAO1, 17: P.putida CFML 90-136, 18: D46, 19: P. putida G168, 20: 96-192, 21: P. libanensis CFML 96-195, 22: Pseudomonas sp. PS6-10, 23: P.putida (Gwose), 24: Lille 40 (9AW), 25: P.fluorescens ATCC 17400, 26: P.fluorescens 1.3, 27: Pseudomonas sp. 96-319, 28: Pseudomonas sp. CHO59, 29: Pseudomonas sp. CIP 75.23

The NCBI Accession Numbers for the 16S rRNA gene sequences of the four isolates (PsS29, PsC132, PsWs140 and PsTp171) determined in this present study are respectively HM 627585, HM 627574, HM 627619 and HM 627603.

In order to investigate whether the four Pseudomonas species produce different pyoverdins, the siderotyping method was used. Table 1 illustrates the different PVD-IEF patterns upon analyzing the culture supernatants of the four strains grown under irondeficient conditions (CAA medium). PsS29 and PsTp171 were characterized by acidic PVD-IEF profiles, with bands ranging between pHi 4.0 and 5.1. PVD-IEF profiles from strain PsWs140 produced two main bands (pHi 8.9 and 7.3) and a minor band with pHi value of 8.5. 
Am. J. Environ. Sci., 8 (2): 143-151, 2012

Table 1: Pyoverdine isoelectrophoretic patterns, ${ }^{59} \mathrm{Fe}$ incorporation and $16 \mathrm{~S}$ rDNA affiliation

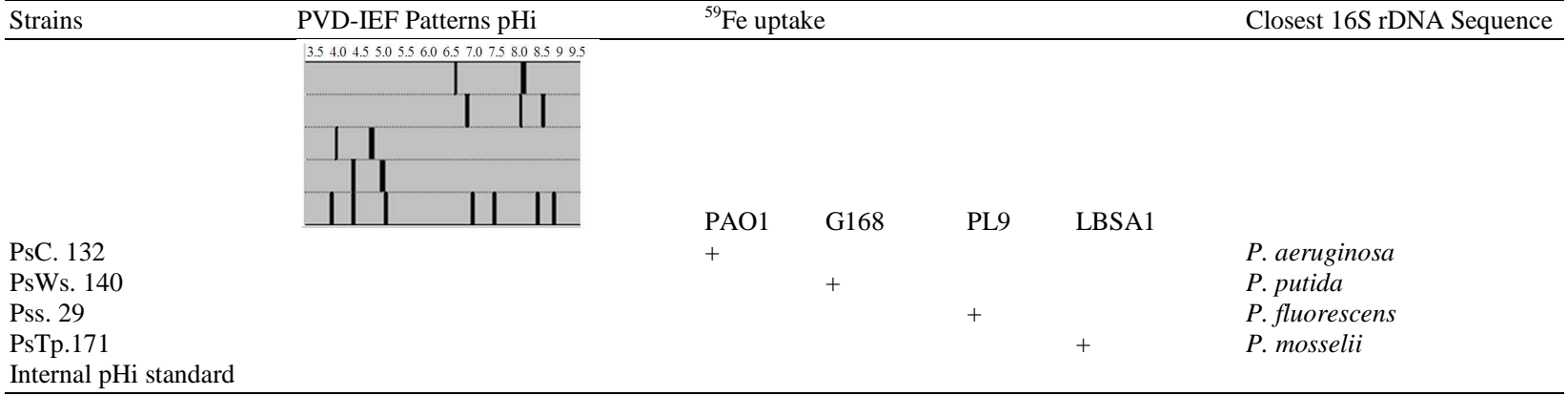

+: homologous incorporation. PAO1: P. aeruginosa, G168: P. putida, PL9: Pseudomonas spp. and LBSA1: Pseudomonas spp

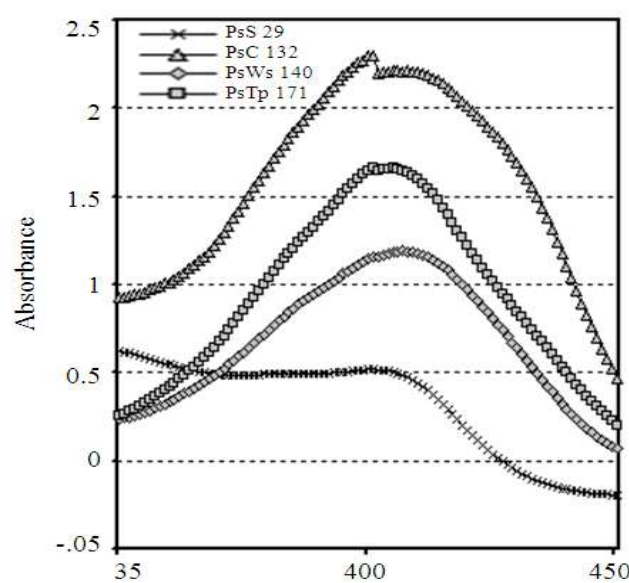

(a)

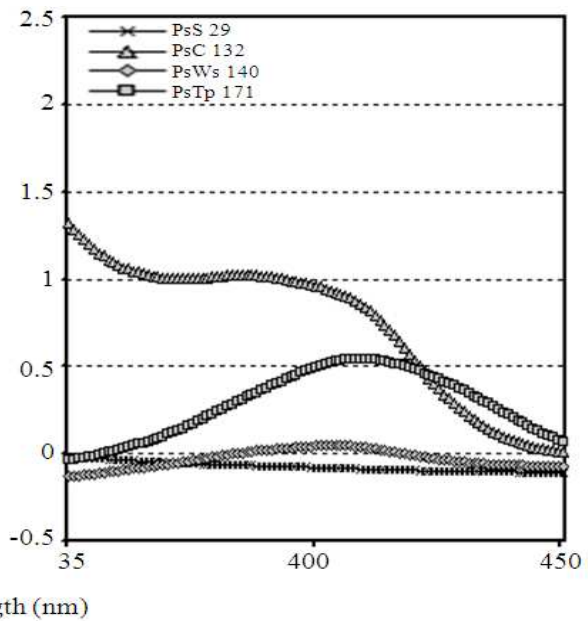

(b)

Fig. 2: Absorption spectra $(350-450 \mathrm{~nm})$ of the un-diluted supernatants from strains: P. fluorescens (PsS29), $P$. aeruginosa (PsC132), P. putida (PsWs140) and P. mosselii (PsTp171) CAA (a) and SM growth media (b)

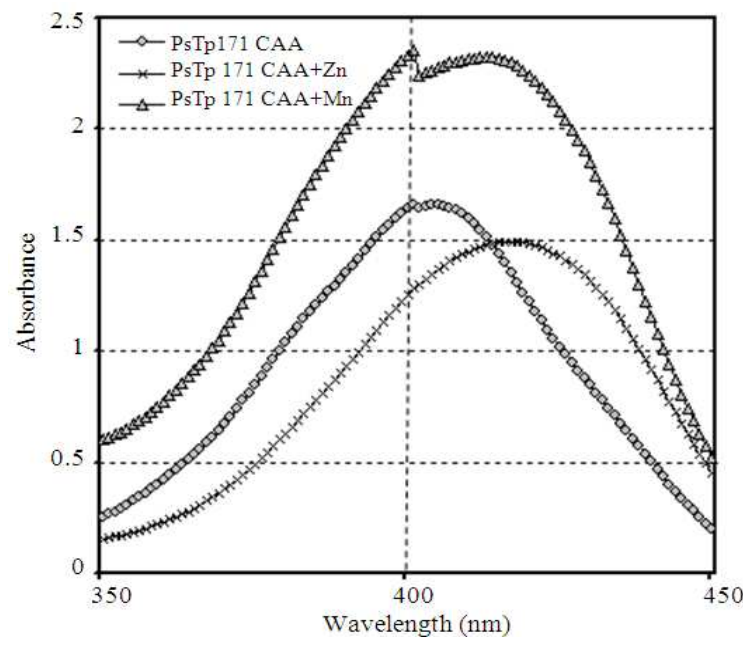

Fig. 3: Absorption spectra $(350-450 \mathrm{~nm})$ of the undiluted clear supernatants from strain $P$. mosselii (PsTp171) grown in indicated media: CAA, $\mathrm{CAA}+\mathrm{Zn}$ and $\mathrm{CAA}+\mathrm{Mn}$
The fourth strain (PsC132) showed two bands with pHi values of 8.5 and 6.9. (Table 1 demonstrate a schematic PVD pattern of the four tested strains).

For the purpose to confirm the classification reached by PVD-IEF characterization, the four strains were analysed for their capacity to incorporate iron under the form of a PVD-iron complex. The strains PsS29, PsTp171, PsWs140 and PsC132 cross-reacted with their own PVDs and with the type strains PL9, LBSA1, G168 and PAO1, respectively (Table 1). As example of homologous and heterologous ${ }^{59} \mathrm{Fe}$ incorporation, the strain PsWs140 showed primordially, a strict specificity of recognition toward its own pyoverdine. Figure 1 showed that the strain PsWs140 exclusively incorporated iron bound to its pyoverdine with a $100 \%$ efficiency (PVD number 30) indeed to the pyoverdine of $P$. putida strain G168 with a $78 \%$ efficiency ( PVD number 19) (Data not shown for other strains). 
Am. J. Environ. Sci., 8 (2): 143-151, 2012
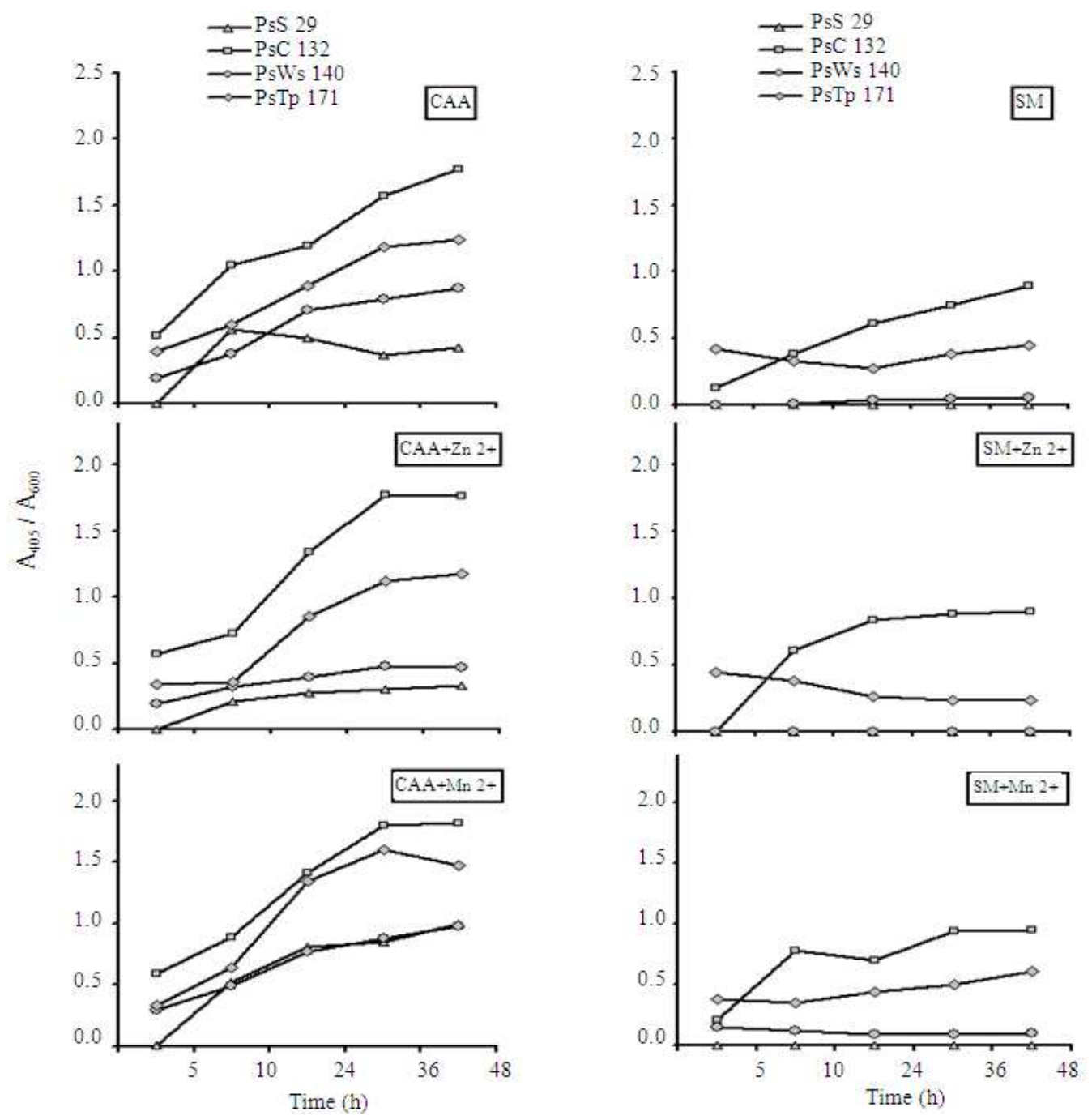

Fig. 4: Influence of supplementation of two trace elements ( $\mathrm{Zn}$ and $\mathrm{Mn}$ ) in CAA (a) and SM (b) media on pyoverdine levels of four different fluorescent Pseudomonas strains $P$. fluorescens (PsS29), P. aeruginosa (PsC132), P. putida (PsWs140) and P. mosselii (PsTp171) at different time intervals. The supernatants were diluted 1:10 in deionised water. Samples were collected in triplicate. $\mathrm{A}_{405} / \mathrm{A}_{600}$ ratio expressed the pyoverdine level (Stintzi et al., 2000)

Strains growth related to pigment synthesis: Spectrophotometric analysis of the un-diluted bacteria supernatant showed an absorption area between 350 and $450 \mathrm{~nm}$ with a sharp peak at about $400 \mathrm{~nm}$ (Fig. 2), characterizing the PVD siderophore type. The maximum absorbance obtained for the strain PsS29 was at $400 \mathrm{~nm}$. The other strains saved the maximum absorbance between 405 and $410 \mathrm{~nm}$.

The determination of siderophore production by the strains used in this study allowed their separation in three types: PsC132 produced the highest siderophore concentration; followed by strains PsTp171 and PsWs140. The least siderophore production was obtained by PsS29 (Fig. 2a). The cells grown on the CAA medium presented siderophore content nearly $2.5-$ fold higher as compared to cells developed in SM medium. No PVD production was detectable for strain the PsS29 in SM medium (Fig. 2b).

Strains grown in CAA medium (Fig. 4a) exhibit a higher PVD level ranging from 1.98-fold (PsC132) to 15.26-fold (PsWs140) compared to SM medium (Fig. $4 \mathrm{~b})$. So, this is due to a significantly higher purity of the CAA medium (lower iron contamination).

Considering the PVD levels related to growth cycle (Fig. 4) the siderophore released by the studied strains in CAA and SM media started after 5 h of incubation 
and increased up to 36-48h then declined afterward. In SM medium, apart PsS29 enable to produce PVD, PsWs140 and PsTp171 strains showed no significant increase in the level of PVD during incubation time (Fig. 4b). The strains were found to produce maximum siderophore quantity during the stationary phase of culture growth (Sharma and Johri, 2003).
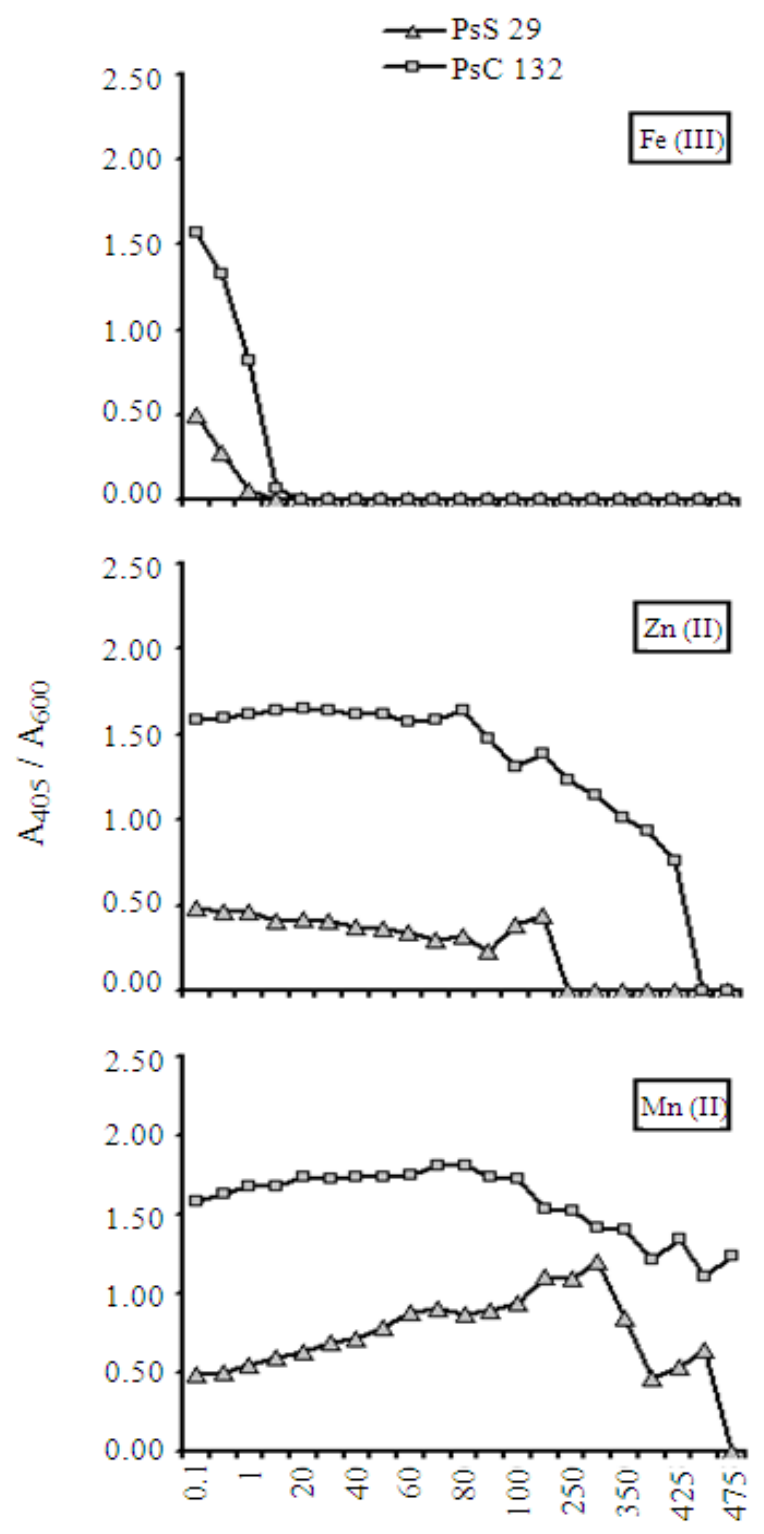

Metal concentration $(\mu \mathrm{M})$

Fig. 5: Dose-response effect of various metals on pyoverdine levels of $P$. fluorescens (PsS29) and $P$. aeruginosa (PsC132) in CAA medium. $\mathrm{A}_{405} / \mathrm{A}_{600}$ ratio expressed the pyoverdine level (Stintzi et al., 2000)
While studying the influence of heavy metals, it was observed that the presence of $\mathrm{Mn}^{2+}$ in the extracellular medium (CAA and SM) promoted significantly PVD production (Braud et al., 2009; Sharma and Johri, 2003). After 48h of growth, PsC132 present a slightly increase in siderophore level in CAA and SM media enriched with Mn supplementation (respectively 1.03-fold and 1.06-fold increase). The presence of $60 \mu \mathrm{M} \mathrm{Mn}{ }^{2+}$ increased PVD production of PsTp171 strain by nearly 1.2 -fold in CAA medium (Fig. 3 and 4a) and nearly 1.37-fold in SM medium (Fig. 4b). However, supplementing CAA and SM media with exogenous $\mathrm{Zn} 2+$, in case of PsTp171 strain, caused a decrease in siderophore levels (respectively 1.05-fold and 1.91-fold decrease) as compared to the control (CAA and SM without metal supplementation) (Fig. 3-5).

\section{DISCUSSION}

The identification based on the $16 \mathrm{~S}$ rDNA sequencing was reconfirmed by using the siderotyping, an easy and powerful method, giving a rapid discrimination between fluorescent pseudomonads producing a particular pyoverdin (Meyer et al., 2002). Therefore, the four studied strains produced compounds belonging to four different siderovars indicating the presence of various pyoverdin structures. The $59 \mathrm{Fe}$ incorporation technique confirms this data. The detection of different siderovars confirms the diversity of the four strains used.

The determination of the absorbance of the clear supernatants obtained from all cultures developed for $48 \mathrm{~h}$ in CAA and SM medium was performed to determine whether the maximum absorbance was at $400 \mathrm{~nm}$. As shown in Fig. 2, the maximum absorbance varied from 400 to $410 \mathrm{~nm}$ which may indicate the diversity of compounds produced by these strains. This multiplicity may be due to the nature and the number of the aminoacyl residues in the peptide moiety (CarrilloCastaneda et al., 2005).

Moreover, the level of siderophore production was compared in the two medium. The pyoverdine production was greater in CAA media. Numerous investigations have shown that the synthesis of PVDs by fluorescent pseudomonads was affected by different environmental factors, notably the chemical nature of the organic carbon and energy source, the degree of aeration of the growth medium, $\mathrm{pH}$, light and trace elements (Gouda and Greppin, 1965; Meyer et al., 1978). Although the different media usually have varied levels of iron contamination, Sharma and Johri (2003) suggested that synthetic media are in all cases better than the complex medium for siderophore production. 
Moreover, Carrillo-Castaneda et al. (2005) demonstrated that iron concentration in the growth medium is an important nutritional factor which determines siderophore biosynthesis.

Strains PsTp171 and PsC132, producing the largest amounts of pyoverdine are isolated respectively from wastewater treatment plant and from compost. Several authors noted the important role of strains isolated from these origins in bioaccumulation of heavy metals. Isolates from complex sources were faced frequently to compete towards micronutrients such as heavy metals via siderophore production (Lovley et al., 1997; Hassen et al., 2001). Hussein et al. (2005) noted that a group of Pseudomonas sp. isolated from the effluent of wastewater treatment plant in western Alexandria, possess the ability to tolerate and to uptake different heavy metals ( $\mathrm{Cu}$ (II), Ni (II)).

Metal ions have definite influence on siderophore production. While $\mathrm{Mn}^{2+}$ increased the siderophore production, $\mathrm{Zn}^{2+}$ decreased this production. This result is consistent with the work of Sayyed et al. (2005), who carried out a notably decrease in the amount of siderophore produced in the case of SM media supplemented with $\mathrm{Zn}^{2+}$. Authors reported that the metal ion can substitute $\mathrm{Fe}^{2+}$ in the intracellular control of siderophoregenesis. In addition, Braud et al. (2010) indicated that besides pyochelin, PVD is able to sequester metals from the extracellular medium of the bacteria, decreasing metal diffusion into the bacteria. In their study, PVD was able to sequester $\mathrm{Al}^{3+}, \mathrm{Co}^{2+}, \mathrm{Cu}^{2+}$, $\mathrm{Eu}^{3+}, \mathrm{Ni}^{2+}, \mathrm{Pb}^{2+}, \mathrm{Tb}^{3+}$ and $\mathrm{Zn}^{2+}$ from the extracellular medium. Baysse et al. (2000) noted that the repression of pyoverdin production by vanadium and explained that uptake of several metals by siderophores was possible.

The dose-response effect of heavy metals on PVD level by the highest (PsC132) and the lowest (PsS29) siderophore producing strains was investigated using the CAA medium growth. Results show that pyoverdine production was inversely related to $\mathrm{Fe}^{3+}$ concentration, whereas growth was directly proportional to iron concentration (Meyer et al., 1978; Visca et al., 1992; Villegas et al., 2002; Manwar et al., 2004). Djibaoui and Bensoltane (2005) observed a complete decline of siderophore production with $200 \mu \mathrm{g} / \mathrm{L}$ of iron as threshold level. This fact reflects the iron requirement for microbiological cellular processes (Sayyed et al., 2005).

On the other hand, concentrations higher then 250 $\mu \mathrm{M}$ of zinc declined the siderophore production. Visca et al. (1992) demonstrated that the pyoverdine production was not affected by metals ( $\mathrm{Zn}(\mathrm{II}), \mathrm{Mo}(\mathrm{VI})$, $\mathrm{Co}(\mathrm{II}), \mathrm{Ni}(\mathrm{II})$ and $\mathrm{Cu}(\mathrm{II})$ ) at concentrations up to $10 \mu \mathrm{M}$ but was repressed at higher concentrations. Therefore, PsC.132 and PsS.29 were highly tolerant to Mn since their pyoverdine production was completely repressed only by high $\mathrm{Mn}$ concentrations (> $475 \mu \mathrm{M})$.

\section{CONCLUSION}

This study revealed by means of the spectrophotometry measurement, classification of environmental strains by levels of siderophore production. Both fluorescent Pseudomonads, $P$. aeruginosa and $P$. mosselii were able to provide higher yields of PVDs especially in CAA medium and in media supplemented with $\mathrm{Mn}^{2+}$. Moreover, at concentration $>20 \mathrm{mM}$ of iron, siderophore biosynthesis is completely quenched.

In culture medium supplemented with zinc, the amount of PVD is lower than that excreted in control medium (no added metal). This result indicates that pyoverdins of studied strains might be able to complex zinc instead of iron. These strains would have the ability to chelae one of essential component and make it inaccessible to other bacteria (competition phenomenon). In addition, this property could be used in the case of decontamination area contaminated with an excess of zinc. Bioremediation using bioaccumulation and chelating of heavy metals contaminating industrial waste may be an alternative processes and/or additives to conventional methods (physical and chemical). Therefore, the ability of fluorescent Pseudomonas, isolated from wastewater treatment plant and from compost, to sequester zinc, point to a unique advantage of these species for divers bioremediation applications.

\section{REFERENCES}

Adarsh, V.K., M. Mishra, S. Chowdhury, M. Sudarshan, A.R. Thakur and S.R. Chaudhuri, 2007. Studies on metal microbe interaction of three bacterial isolates from east Calcutta Wetland. J. Biol. Sci., 7: 80-88.

Ambrosi, C., L. Leoni and P. Visca, 2002. Different Responses of Pyoverdine Genes to Autoinduction in Pseudomonas aeruginosa and the Group Pseudomonas fluorescens-Pseudomonas putida. Applied Environ. Microbiol., 68: 4122-4126. DOI: 10.1128/AEM.68.8.4122-4126.2002

Baysse, C., D.D. Vos, Y. Naudet, A. Vandermonde, U. Ochsner, and J.M. Meyer et al., 2000. Vanadium interferes with siderophores-mediated iron uptake in Pseudomonas aeruginosa. Microbiology, 146: 2425-2434. PMID: 11021919

Braud, A., F. Hoegy, K. Jezequel, T. Lebeau and I.J. Schalk, 2009. New insights into the metal specificity of the Pseudomonas aeruginosa pyoverdine-iron uptake pathway. Environ. Microbiol., 11: 1079-1091. DOI: 10.1111/j.14622920.2008.01838.x 
Braud, A., V. Geoffroy, F. Hoegy, G.L.A. Mislin and I.J. Schalk 2010. Presence of the siderophores pyoverdine and pyochelin in the extracellular medium reduces toxic metal accumulation in Pseudomonas aeruginosa and increases bacterial metal tolerance. Environ. Microbiol. Reports, 2: 419-425. DOI: $\quad 10.1111 / \mathrm{j} .1758$ 2229.2009.00126.x

Braun, V. and M. Braun, 2002. Iron transport and signalling in Escherichia coli. FEBS Lett., 529: 7885. PMID: 12354617

Bruins, M.R., S. Kapil and F.W. Oehme 2000. Microbial resistance to metals in the environment. Ecotoxicol. Environ. Safety, 45: 198-207. DOI: 10.1006/eesa.1999.1860

Canovas, D., I. Cases and V.D. Lorenzo, 2003. Heavy metal tolerance and metal homeostasis in Pseudomonas putida as revealed by complete genome analysis. Environ. Microbiol., 5: 12421256. DOI: $10.1111 /$ j.1462-2920.2003.00463.x

Carrillo-Castaneda, G., J.J. Munoz and J.R. PeraltaVidea, 2005. A spectrophotometric method to determine the siderophore production by strains of fluorescent Pseudomonas in the presence of copper and iron. Microchem. J., 81: 35-40. DOI: 10.1016/j.microc.2005.01.018

Cornelis, P. and M. Matthijs, 2002. Diversity of siderophore-mediated iron uptake systems in fluorescent pseudomonads: not only pyoverdines. Environ. Microbiol., 4: 777-923. DOI: 10.1046/j.1462-2920.2002.00369.x

Olmo, A.D., C. Caramelo and C. SanJose, 2003. Fluorescent complex of pyoverdin with aluminum. J. Inorg. Biochem., 97: 384-387. DOI: 10.1016/S0162-0134(03)00316-7

Villegas, M.E.D.D., P. Villa and A. Frias 2002. Evaluation of the siderophores production by Pseudomonas aeruginosa PSS. Rev. Latinoam. Microbiol., 44: 112-117. PMID: 17061484

Djibaoui, R. and A. Bensoltane, 2005. Effect of iron and growth inhibitors on siderophores production by Pseudomonas fluorescens. Afr. J. Biotechnol., 4: 697-702. ISSN: 1684-5315

Fuchs, R., M. Schafer, V. Geoffroy and J.M. Meyer, 2001. Siderotyping-a powerful tool for the characterization of pyoverdines. Curr. Top. Med. Chem., 1: 31-57. DOI: 10.2174/1568026013395542

Gouda, S. and H. Greppin, 1965. Biosynthese pigmentaire chez Pseudomonas fluorescens en fonction de la concentration du substrat hydrocarbone ou amine. Arch Scienc, Geneve, 18: 716-721.
Kumar, A., S. Gaind and L. Nain, 2008. Evaluation of thermophilic fungal consortium for paddy straw composting. 19: 395-402. DOI: 10.1007/s10532007-9145-3

Hassen, A., K. Belguith, N. Jedidi, A. Cherif and M. Sherif et al., 2001. Microbial characterization during composting of municipal solid waste. Bioresource Technol., 80: 217-225. DOI: 10.1016/S0960-8524(01)00065-7

Henry, M.B., J.M. Lynch and T.R. Fermor, 1991. Role of siderophores in the biocontrol of Pseudomonas tolaasii by fluorescent pseudomonad antagonists. J. Applied Bacteriol., 70: 104-108. DOI: 10.1111/j.1365-2672.1991.tb04434.x

Hussein, H., S. Farag, K. Kandil and H. Moawad, 2005. Tolerance and uptake of heavy metals by Pseudomonads. Process Biochem. 40: 955-961. DOI: $10.1016 /$ j.procbio.2004.04.001

Lovley, D.R. and J.D. Coates, 1997. Bioremediation of metal contamination. Curr. Opin. Biotechnol., 8: 258-289. DOI: 10.1016/S0958-1669(97)80005-5

Manwar, A.V., S.R. Khandelwal, B.L. Chaudhari, J.M. Meyer and S.B. Chincholkar, 2004. Siderophore production by a marine Pseudomonas aeruginosa and its antagonistic action against phytopathogenic fungi. Applied Biochem. Biothechnol., 118: 243251. DOI: $10.1385 / \mathrm{ABAB}: 118: 1-3: 243$

Meyer, J.M., 2000. Pyoverdines: Pigments, siderophores and potential taxonomic markers of fluorescent Pseudomonas species. Arch. Microbiol., 174: 135-142. DOI: $10.1007 / \mathrm{s} 002030000188$

Meyer, J.M. and M.A. Abdallah, 1978. The fluorescent pigment of pseudomonas fluorescens: Biosynthesis, purification and physicochemical properties. J. Gen. Microbiol., 107: 319-328. DOI: 10.1099/00221287107-2-319

Meyer, J.M., V.A. Geoffroy, N. Baida, L. Gardan and D. Izard et al., 2002. Siderophore typing, a powerful tool for the identification of fluorescent and nonfluorescent pseudomonads. Applied Environ. Microbiol., 68: 2745-2753. DOI: 10.1128/AEM.68.6.2745-2753.2002

Mureseanu, M., G. Renard, A. Galarneau and D.A. Lerner, 2003. A demonstration model for a selective and recyclable uptake of metals from water: $\mathrm{Fe}(\mathrm{III})$ ions complexation and release by a supported natural fluorescent chelator. Talanta, 60: 515-522. DOI: 10.1016/S0039-9140(03)00103-6

Winkelmann, G., D.V.D. Helm and J.B. Neilands, 1987. Iron Transport In Microbes, Plants and Animals. 1st Edn., VCH, Weinheim, ISBN-10: 3527266852 pp: 533. 
Parker, D.L., G. Sposito and B.M. Tebo, 2004. Manganese(III) binding to a pyoverdine siderophore produced by a manganese(II)-oxidizing bacterium. Geochim. Cosmochim. Acta, 68: 4809-4820. DOI: 10.1016/j.gca.2004.05.038

Saidi, N., S. Kouki, F. M'hiri, M.R. Hajlaoui and M. Mahrouk et al., 2009. Characterization and selection of Bacillus sp. Strains, effective biocontrol agents agains Fuzarium oxysporum f.sp.radicis-lycopercisi, the causal agent of Fusarium crown and root root rot in tomato. Annals Microbiol., 59: 191-198.

Sayyed, R.Z., M.D. Badgujar, H.M. Sonawane, M.M. Mhaske and S.B. Chincholkar, 2005. Production of microbial iron chelators (siderophores) by fluorescent Pseudomonads. Indian J. Biotechnol., 4: 484-490.

Sharma, A. and B.N. Johri, 2003. Combat of irondeprivation through a plant growth promoting fluorescent Pseudomonas strain GRP3A in mung bean (Vigna radiata L. Wilzeck). Microbiol. Res., 158: 77-81. DOI: 10.1078/0944-5013-00182

Shinozaki-Tajiri, Y., Y. Akutsu-Shigeno, T. NakajimaKambe, S. Inomata and N. Nomura et al., 2004. Matrix Metalloproteinase-2 Inhibition and $\mathrm{Zn} 2+-$ chelating activities of pyoverdine-type siderophores. J. Biosci. Bioeng., 97: 281-283. DOI: 10.1263/jbb.97.281
Stintzi, A., C. Barnes, J. Xu and K.N. Raymond, 2000. Microbial iron transport via a siderophore shuttle: A membrane ion transport paradigm. Proc. Natl. Acad. Sci. U.S.A., 97: 10691-10696. DOI: 10.1073/pnas.200318797

Stuczynski, T.I., G.W. McCarty and G. Siebielec, 2003. Response of soil microbiological activities to Cadmium, Lead and Zinc salt amendments. J. Environ. Qual., 32: 1346-1355. PMID: 12931890

Teitzel, G.M., A. Geddie, S.K.D. Long, M.J. Kirisits and M. Whiteley et al., 2006. Survival and growth in the presence of elevated copper: Transcriptional profiling of copper-stressed Pseudomonas aeruginosa. J. Bacteriol., 188: 7242-7256. DOI: 10.1128/JB.00837-06

Visca, P., G. Colotti, L. Serino, D. Verzili and N. Orsi et al., 1992. Metal regulation of siderophore synthesis in Pseudomonas aeruginosa and functional effects of siderophore-metal complexes. Applied Environ. Microbiol., 58: 2886-2893.

Wahyudi, A.T., Indri, R. and Giyanto, 2011. Screening of Pseudomonas sp. Isolated from rhizosphere of soybean plant growth promoter and biocontrol agent. Am. J. Agric. Biol. Sci., 6: 134-141.

Yang, C.H., Y.C. Huang and C.Y. Chen, 2009. Degradation of rutin by thermoactinomyces vulgaris and other thermophilic compost isolates. J. Agric. Food Chem., 57: 5095-5099. DOI: 10.1021/jf900617z 